\title{
EFICIENCIA DEL GASTO E IMPACTO ECONÓMICO DEL SEGURO POPULAR DE SALUD: EL CASO DE TABASCO
}

EFFICIENCY OF PUBLIC SPENDING AND ECONOMIC IMPACT OF “SEGURO POPULAR DE SALUD” THE CASE OF TABASCO

\begin{tabular}{c|c} 
Noé Arón Fuentes Flores \\
$\begin{array}{c}\text { Colegio de la Frontera Norte, México } \\
\text { afuentes@colef.mx }\end{array}$ & $\begin{array}{c}\text { Rosa Isabel Magadán Vega } \\
\text { Colegio de la Frontera Norte, México } \\
\text { mave_300@yahoo.com.mx }\end{array}$
\end{tabular}

\section{RESUMEN}

El principal propósito del estudio es presentar una estrategia metodológica que analiza los impactos económicos del Sistema de Protección Social en Salud (SPSS) en el estado de Tabasco, a través de la vinculación de la Cuenta Satélite en Salud y la Matriz Insumo Producto del estado. Así, el objetivo general del análisis consiste en valorar el alcance de los impactos, es decir, definir su intensidad según su efecto sobre los indicadores de ingreso, producción y empleo en el estado.

Palabras claves: análisis de impacto, Sistema de Protección Social en Salud, ingreso, producción y empleo.

Clasificación JEL: E10, E27 


\section{ABSTRACT}

The main purpose of the study is to present a methodological strategy to analyze the economic impacts of the System of Social Protection in Health (SPSS) in the State of Tabasco, linking the Satellite Account in Health and the Input Output Table. Thus the overall objective of the analysis is on assessing the extent of the impacts, in other words, defining its intensity according to their effect on the indicators of income, production and employment in the State.

Keywords: Impact Analysis, System of Social Protection in Health, Income, Production and employment. 


\section{INTRODUCCIÓN}

La evaluación de los impactos de las políticas de salud implica contar con elementos concretos y objetivos que permitan mejorar los instrumentos, lo que permite introducir los ajustes necesarios para garantizar el máximo bienestar de la población. La actual política en México está dirigida a colaborar con el beneficio social, orientado a mejorar la eficiencia y equidad en la provisión de servicios médicos y contribuir con el desarrollo económico de las regiones; lo anterior supone la necesidad de contar con información y herramientas apropiadas para el proceso evaluativo de las políticas de salud y sus instrumentos.

Entre los años 1994 y 2000 se inicia en el país un periodo de reforma del sector salud, en el que se expone la necesidad de instrumentar políticas sustentables que amplíen la cobertura de los servicios y rectifiquen la falta de precisión en la definición de las responsabilidades de cada orden de gobierno (Ángeles, et al. 2011). Bajo estas primicias, en 2001 se crea el Sistema de Protección Social en Salud (SPSS) o Seguro Popular, el cual fue institucionalizado en 2004 como un eje rector del nuevo paradigma de salud pública en México. A once años de su creación, el SPSS tiene presencia en toda la república, hecho que beneficia el análisis global y regional de su desarrollo.

A causa de la naturaleza innovadora, el SPSS ha sido objeto de diversas investigaciones evaluativas con diferentes perspectivas y dimensiones, que analizan el desarrollo interno del Seguro Popular y los impactos del gasto en el ámbito social y de salud. Sin embargo, el presente estudio pretende aportar una perspectiva metodológica que amalgama dos instrumentos evaluativos que miden y cuantifican el nivel y dirección de impactos que ejerce el SPSS en el sistema de salud y la economía regional.

Ahora bien, la descentralización operativa provee independencia en las acciones de cada región y, en consecuencia, resultados desiguales; el estado de Tabasco, además de ser una entidad pionera en la introducción del Seguro Popular, se ha caracterizado por ser un caso atípico en la evolución del gasto presupuestal y progreso en la cobertura. Si bien Tabasco posee características especiales como objeto de estudio, provee 
un instrumento de análisis específico para una evaluación de impactos integral: La Cuenta Satélite en Salud.

La CSS-T amplía la capacidad analítica de un sector para proporcionar información intrasectorial, al medir diversas variables como producción, consumo intermedio o valor agregado bruto, al mismo tiempo vincula los flujos monetarios con el análisis de gasto por los agentes (United Nations et al., 2001). En tanto que el estudio de la incidencia económica, el marco intersectorial o insumo-producto nos permite medir los efectos directos, indirectos e inducidos por un cambio en la demanda de servicios de salud (Hernández, 2004). En resumen, el modelo metodológico permite observar los impactos en los ámbitos sectorial y regional, al relacionar los resultados en estos dos recortes espaciales, que deriva en un análisis de la cadena de impactos del Seguro Popular en Tabasco.

El objetivo es, por lo tanto, presentar un análisis de la efectividad de la erogación pública y privada en atención médica en el estado y, adicionalmente, exponer las cuestiones metodológicas del impacto económico, tratando de valorar si éste es elevado o no, según su efecto sobre los indicadores estatales de ingreso, producción y empleo.

El artículo se organiza de la siguiente manera: en la segunda sección se presentan brevemente los antecedentes del seguro popular en el estado de Tabasco, destacándose que es la entidad federativa que posee el más alto nivel de gasto en salud y, además, de registrar el SPSS, a nivel nacional, la mayor proporción de la población abierta. En la sección tres se muestran las diversas metodologías empleadas para evaluar la importancia relativa del sector salud en el conjunto de la economía. Se resalta que se han utilizado, tradicionalmente, cuatro metodologías para elaborar los impactos económicos del sector: las Cuentas Satélite (CS), la matriz de insumo-producto (MIP), el análisis beneficio-costo (ACB), así como los análisis sectoriales y regionales (ASyR). En la cuarta se exhibe la matriz de insumo producto de Tabasco para 2003, realizada por iniciativa del gobierno estatal, y en la que se reconocen y agrupan 17 actividades principales. En la quinta se presenta el sistema de cuenta satélite de Tabasco de 2003, que proporciona información intrasectorial, se mide especialmente el valor agregado bruto de la salud, el consumo intermedio del sector y el 
producto interno bruto, así como la vinculación de los flujos monetarios con el análisis de gasto por agentes, lo que permite crear las bases de indicadores y análisis del sector. En la sección seis se describe, según la Cuenta Satélite de Tabasco, quién gasta, en qué actividades se gasta, qué productos se compran y cómo se financian los gastos de salud. En la siete se muestran los impactos por sector económico debido a cambios exógenos en los componentes de la demanda final (gasto de gobierno en el mencionado sector) y se calculan tantos multiplicadores (producto, ingreso y empleo) como sectores haya incluidos en la matriz de transacciones intersectoriales de Tabasco. Finalmente, en la sección ocho se presentan las principales conclusiones del estudio.

\section{ANTECEDENTES DEL SPSS EN TABASCO}

La Secretaría de Salud (SS) concluyó "a partir de la Encuesta nacional de ingreso-gastos y la Encuesta de satisfacción de los servicios de salud 2000, no están en las referencias poner o eliminar que el contar con un seguro médico es un factor que reduce el riesgo de empobrecer y, en consecuencia, uno de cada cuatros mexicanos pospuso su atención médica por problemas económicos" (SSA, 2002:65); el principal problema del Sistema de Salud es la falta de cobertura y equidad (o justicia), esto se traduce en gastos de salud excesivos - catastróficos y empobrecedores - que resultan en la disminución de acceso y calidad en los servicios de salud e ineficiencia en el empleo de recursos. En consecuencia, la falta de protección financiera en salud afecta directamente a los hogares que no están cubiertos por ningún órgano público o privado (SSA, 2002).

Asimismo, el censo de población y vivienda de 2000 (INEGI, 2000), reportó que $57.8 \%$ de la población en el país no poseía ningún tipo de seguro, tal hecho atenta contra la economía familiar. Este segmento de la población se coloca en una situación altamente vulnerable, ya que desembolsa directamente sus ingresos para atender su salud, por lo tanto el gasto de bolsillo ejercido es destinado a la compra de medicamentos y consultas médicas, lo que afecta a las familias de bajos ingresos. Queda claro que el gasto de bolsillo ejerce mayor impacto en las familias en situación económica vulnerable 
en comparación de aquellas familias con mayores recursos, de acuerdo con la Secretaría de Salud $85 \%$ de las familias en situación económicamente vulnerable se empobrecen a raíz de este gasto de bolsillo y no están aseguradas (SSA, 2002). Frente a este desafío, en 2001 se propuso la integración de un programa piloto denominado Seguro Popular de Salud, “(...)ante la necesidad de proveer una alternativa de atención a la salud, mediante un esquema de aseguramiento público, para aquella población que no contaba con acceso a servicios de salud(...)" (CNPSS, 2010:11). En abril de 2003 bajo la reforma de la Ley general de salud fue institucionalizado a nivel federal y en enero de 2004 empezó a operar formalmente como el Sistema de Protección Social en Salud.

Desde sus inicios como programa piloto propuso el objetivo de "brindar protección financiera a todos los mexicanos (...) ofreciendo una opción de aseguramiento público en materia de salud a familiar y los ciudadanos por su condición laboral y socioeconómica no son derechohabientes de las instituciones de seguridad social" (SSA, 2002:66). Sujeto a este objetivo principal se desprenden cuatro objetivos específicos: reducir el gasto privado de bolsillo, fomentar atención oportuna en salud, fortalecer el sistema de salud y, finalmente, contribuir a superar inequidades y rezagos en la distribución del gasto entre entidades federativas con diferentes niveles de desarrollo en materia de salud.

Al poseer una desconcentración operativa el sistema tiende a particularizarse, es decir, tiende a poseer características de desarrollo acordes a la región donde opera; los factores sociales, políticos, económicos y de salud crean un contexto regional distinto, además que el periodo de incorporación podría influir en el desarrollo del sistema. La presente evaluación se acota espacialmente en el estado de Tabasco ¿por qué este recorte espacial?, primero, Tabasco puede ser nombrado como uno de los precursores del programa piloto del Seguro Popular de Salud; desde la incorporación del estado al proyecto en el 2001 muestra cualidades distintas, es decir, presenta una madurez distinta del SPSS a nivel nacional, así mismo posee el más alto nivel de gasto en salud ${ }^{1}$, al destinar $6.72 \%$

\footnotetext{
${ }^{1}$ Gastos en salud: engloban los gastos monetarios en cuidados de la salud realizados por el hogar. Los rubros considerados son los siguientes: servicios médicos durante el parto, servicios médicos y medicamentos durante el embarazo, consulta externa (no hospitalaria
} 
de su PIB (SSA, 2008), además de poseer, nacionalmente, la más alta proporción de población abierta afiliada al SPSS (Rangel y Martínez, 2007).

Así pues, el estado reúne la características para presentarse como un caso distinto en el país debido a su cobertura en SPSS que está en consonancia con la captación de cuotas familiares del régimen contributivo del sistema, Tabasco es la única entidad a nivel nacional que posee la captación más alta, a saber: 148,566,347.0 (CNPSS,2009:28).

Finalmente, se delimita un caso atípico que desarrolla una política pública que ha tomado una gran importancia en el contexto social y de salud, por lo tanto se plantea un estudio que analice los impactos económicos que pueda sufrir el estado.

\section{ENFOQUES DE MEDICIÓN DE LA INCIDENCIA ECONÓMICA EN EL SECTOR SALUD}

Evaluar la incidencia económica del sector de la salud constituye una labor compleja y difícil, tanto por la falta de rigor y precisión conceptual existente al respecto, así como por la abundancia de los flujos reales y financieros de forma transversal en el sistema económico. Por ello, resulta necesario detallar, en la medida de lo posible, qué se intenta medir, con qué instrumentos se cuenta y cómo se procede a la cuantificación de su impacto sobre el desarrollo económico.

En términos generales, los efectos directos e indirectos del sector de la salud hacen referencia a su incidencia sobre el resto del sistema económico de un área geográfica determinada (local, regional, nacional, internacional), de igual forma se derivan los gastos realizados, el financiamiento recibido y el funcionamiento del sector (operación, actividades, etc.). Se admiten tres niveles de análisis: macro (enfoque general), micro y mesoeconómico (enfoque parcial) (Heinemann, 1998; Halba, 1997).

De manera general se trata de evaluar la importancia relativa del sector de la salud en el conjunto de la economía regional. En tanto,

ni embarazo), control de peso, atención hospitalaria, medicamentos sin receta y material de curación, medicina alternativa, aparatos ortopédicos y terapéuticos y seguro médico. 
el análisis microeconómico investiga comportamientos (de hogares, empresas, Estado) y decisiones sectoriales, así como sus posibles interrelaciones. Por último, el análisis mesoeconómico se inspira en la economía industrial y analiza el sector de la salud en términos de cadenas de producción (filière) (Moati, 1990).

La medición requiere que, en función del nivel de análisis o del enfoque seleccionado, se identifiquen correctamente: los gastos realizados, el financiamiento percibido y su procedencia, el valor de la producción de bienes y servicios, la cantidad de factores utilizados en ella, el valor agregado creado, las importaciones y exportaciones efectuadas y los costes y beneficios de su actividad o de un programa salud. En suma, estadísticas capaces de proporcionar datos regulares, sistemáticos, fiables, comparables en el tiempo y en el espacio (dentro y entre diferentes sistemas económicos) y que, además, puedan ser encuadrados en un marco metodológico reconocido internacionalmente (Otero et al., 2000:15), basado en el uso de la lógica del análisis económico. La literatura relacionada con este campo ha venido utilizando, tradicionalmente, seis instrumentos para elaborar las magnitudes económicas del sector salud: las Cuentas Satélite, la Matriz de Insumo-Producto (MIP), el Análisis Beneficio Coste (ACB), así como los análisis sectoriales y regionales. Sus objetivos y principales características están en el cuadro 1.

\section{Cuadro 1}

Características de los principales instrumentos de incidencia económica

\begin{tabular}{|c|c|}
\hline $\begin{array}{c}\text { Cuenta } \\
\text { Satélite (CS) }\end{array}$ & $\begin{array}{l}\text { - Agrupa los datos en unidades monetarias y magnitudes no monetarias. } \\
\text { - Facilita el análisis de las relaciones económicas en el entramado } \\
\text { económico del sector salud y el resto de la economía. } \\
\text { - Permite calcular razones e indicadores que relacionen la importancia } \\
\text { económica del ámbito objeto de análisis (turismo, salud, educación) } \\
\text { con la de economía nacional o regional. Por tanto, puede ser utilizada } \\
\text { para medir el impacto de las actividades de atención de la salud. }\end{array}$ \\
\hline
\end{tabular}




\begin{tabular}{|c|c|}
\hline $\begin{array}{l}\text { Análisis } \\
\text { Insumo- } \\
\text { Producto } \\
\text { (MIP) }\end{array}$ & $\begin{array}{l}\text { - Da una visión desagregada de la actividad económica en cada industria } \\
\text { o rama de actividad y de la interdependencia entre todas ellas. } \\
\text { - Da una percepción precisa de la incidencia económica de la sanidad y } \\
\text { de sus componentes sectoriales, a través de los coeficientes técnicos. } \\
\text { - Propone una contabilización que no aísla la economía de la salud del } \\
\text { resto del sistema económico. } \\
\text { - Permite evaluar la incidencia de una variación exógena de la demanda } \\
\text { final sobre el sistema productivo. Por tanto, puede ser utilizado para } \\
\text { simular la de eventos y medidas de política económica de la salud. }\end{array}$ \\
\hline $\begin{array}{c}\text { Análisis } \\
\text { Beneficio- } \\
\text { Coste (ACB) }\end{array}$ & $\begin{array}{l}\text { Útil para respaldar decisiones sobre evaluación de proyectos o } \\
\text { programas de salud, respecto a la utilización alternativa de recursos. } \\
\text { - Proporciona un criterio de evaluación global de un proyecto público, en } \\
\text { términos de eficiencia y equidad, y una visión global de los efectos de } \\
\text { las actividades sanitarias sobre la localidad. } \\
\text { - Permite tratar de forma adecuada los beneficios externos asociados a } \\
\text { un proyecto. } \\
\text { - Clasifica los efectos inducidos en categorías bien definidas de costes y } \\
\text { beneficios e incluyen tanto las incidencias indirectas como las } \\
\text { intangibles cualitativas), difíciles o imposibles de monetizar. }\end{array}$ \\
\hline $\begin{array}{c}\text { Análisis } \\
\text { Sectoriales y } \\
\text { Análisis } \\
\text { Regional } \\
\text { (ASyR) }\end{array}$ & $\begin{array}{l}\text { - Facilita datos detallados sobre gastos, consumos, presupuestos, } \\
\text { etcétera; vinculados a la salud. } \\
\text { Enriquecen y mejoran las cuentas de la Contabilidad Nacional, al } \\
\text { recoger informaciones cuantitativas y cualitativas específicas al sector } \\
\text { o área geográfica objeto de análisis. }\end{array}$ \\
\hline
\end{tabular}

Fuente: Villalba Caballo (2002).

Los dos primeros enfoques adoptan una perspectiva macroeconómica que permite desagregar los flujos económicos de todo el sector de la salud de un país o incluso de una región; la Cuenta Satélite y la Matriz de Insumo-Producto permiten construir un sistema de cuentas económicas, apoyándose en el principio de la contabilidad por partida doble, y evaluar la incidencia económica ex-post del sector salud desde el punto de vista de la oferta (producción), la demanda (consumo público 
y privado, formación bruta de capital fijo, exportaciones netas) y su financiación.

Adicionalmente, la Cuenta Satélite agrupa los datos en unidades monetarias y no monetarias y permite calcular coeficientes e indicadores de la participación de los servicios de salud en el conjunto de la economía; en tanto que el marco contable de la Matriz de Insumo-Producto posibilita la elaboración de complejos modelos económicos de simulación del impacto bajo diversos escenarios. Ambas pueden ser utilizadas para valorar la incidencia de los impactos de la salud.

El tercer instrumento ofrece, en cambio, un enfoque parcial de cuentas o simplemente de indicadores del sector salud, con una dimensión subregional (Andreff, 2001). Aunque de una forma más amplia, el análisis beneficio coste (ACB) constituye un método flexible que permite identificar y evaluar las repercusiones socioeconómicas de proyectos y programas públicos de salud, con el objeto de determinar su (no) justificación desde un punto de vista económico (Késenne, 2001).

En particular, el análisis beneficio coste destaca, por una parte, su utilidad para respaldar decisiones sobre evaluación de proyectos o programas de salud respecto a la utilización alternativa de recursos y, por la otra, proporciona un criterio de evaluación global de un proyecto público, en términos de eficiencia y equidad, y una visión global de los efectos de las actividades sanitarias sobre la localidad. Más aún, este enfoque permite tratar de forma adecuada los beneficios externos asociados a un proyecto de salud y clasifica los efectos inducidos en categorías bien definidas de costes y beneficios, e incluyen tanto las incidencias indirectas como las intangibles cualitativas, difíciles o imposibles de monetizar.

En síntesis, para evaluar la incidencia económica del sector de la salud en el caso de Tabasco usamos los dos primeros enfoques debido a que adoptan una perspectiva macroeconómica, intersectorial, intrasectorial y regional. Los análisis sectoriales y regionales completan, enriquecen y mejoran las magnitudes que proporcionan las cuentas nacionales del sector salud. Suministran informaciones desagregadas y detalladas (cuantitativas y cualitativas), específicas del sector (financiamiento, gestión, fiscalidad, mercados de asistencia médica, etc.) o de la economía 
regional o local que tratan de analizar (subsidios y presupuestos, cotizaciones y licencias, empleos en el sector, etc.).

Los factores que caracterizan el caso del SPSS-Tabasco, como un fenómeno atípico, son los mismos que definen la perspectiva que adopta el estudio, pues diseñar un análisis sectorial y regional facilita exponer detalladamente los datos sobre los gastos, consumos, presupuestos, etcétera; vinculados al sector salud. Así, el presente diseño metodológico tiene la flexibilidad de vincular el análisis del Seguro Popular, el sector salud y los demás sectores de la economía regional, lo que permite observar los impactos en cada uno de los niveles. De forma general este tipo de estudios enriquece y mejora las cuentas de la contabilidad nacional al recoger informaciones cuantitativas y cualitativas específicas al sector o área geográfica objeto de análisis.

\section{MATRIZ DE INSUMO-PRODUCTO DE TABASCO}

La salud representa un factor imprescindible para el desarrollo de una región; sin embargo, identificar los efectos reales de la salud en el sistema económico es difícil de determinar debido a su naturaleza transversal; por consiguiente, evaluar el impacto económico del sector de la salud en Tabasco constituye una labor compleja, una tarea nada fácil. Por ello, resulta necesario precisar cómo proceder a la cuantificación de su incidencia directa e indirecta sobre el producto, ingreso y empleo.

Desde una perspectiva conceptual, la cuantificación de los efectos multiplicadores del sector de la salud requiere la distinción entre los denominados impactos directos, indirectos e inducidos. Los primeros son los que se producen sobre los sectores de actividad que atienden directamente la demanda de salud. Los impactos indirectos serían aquellos que tienen lugar a través de la cadena de relaciones intersectoriales que se originan a partir del efecto directo, es decir, los impactos indirectos incluyen el efecto del gasto inicial sobre los sectores que suministran insumos intermedios y, a su vez sobre la cadena de proveedores de estos últimos. Finalmente, los impactos inducidos tienen su origen en el gasto del ingreso que se genera como consecuencia de los impactos directos e indirectos (Fuentes y Cárdenas, 2010). 
Estos conceptos han sido desarrollados ampliamente en el marco analítico de interdependencias sectoriales o Matriz de Insumo-Producto. Tabasco cuenta con una matriz, MIP-T, realizada por iniciativa del Gobierno del Estado, cuya construcción se basa en el método directo de recopilación de acuerdo con las recomendaciones del Sistema de Cuentas Nacionales de 1993 (SCN) de Naciones Unidas. ${ }^{2}$ En ella se reconocieron y agruparon 16 actividades principales (Armenta, 2007).

En el cuadro 2 se muestra una versión modificada de la tabla intersectorial de Tabasco para 2003, compuesta por una serie de cuadros que describen las diversas transacciones de la economía estatal, y que conforman el proceso productivo, la función del consumo, la función de la acumulación del capital físico, las ventas y las compras del exterior y la generación del ingreso. Estas transacciones se enmarcan en el equilibrio general dado por la igualdad de la oferta y la demanda y la consistencia de los precios pagados por los compradores y los precios recibidos por los productores (descontando impuestos sobre los productos y márgenes de comercio y transporte de carga).

En la MIP-T los aspectos analíticos centrales son los flujos de producción, particularmente los intersectoriales, así como la interrelación de la demanda y oferta intermedia. Bajo esta lógica la matriz se puede interpretar o leer por filas y por columnas, la lectura por fila muestra el destino de los bienes y servicios, ya sea hacia la demanda intermedia (productos utilizados como insumos) o hacia la demanda final. La lectura por columna muestra la demanda de bienes y servicios por parte de las actividades económicas.

\footnotetext{
${ }^{2}$ El Sistema de Cuentas Nacionales representa el registro sistemático de todos los flujos e inventarios de la economía en un esquema integrado, coherente e interrelacionado de cuentas, transacciones y unidades institucionales (Eurostat et al. 1993).
} 


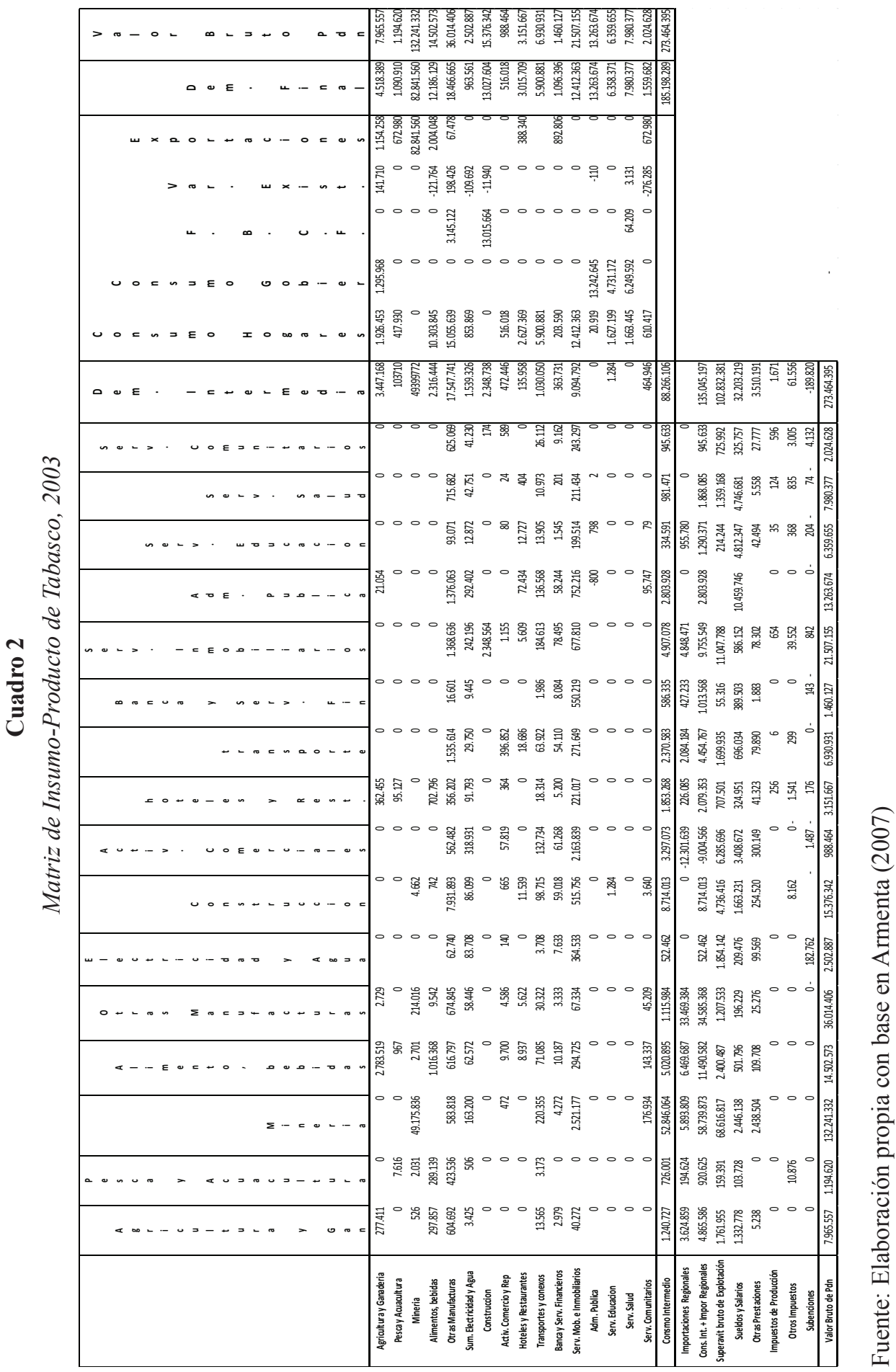

Enero a Junio de 2013 - Págs: 63-98 
Si se establece un corte horizontal en la línea divisoria de los cuadrantes I y II las filas de la parte superior reflejan la oferta de productos, ${ }^{3}$ y las de la parte inferior la oferta de factores productivos utilizados por cada agente económico, oferta que puede ser de origen nacional o extranjero (importaciones). ${ }^{4}$ Por otro lado, las columnas detallan las compras o la demanda por parte de los agentes. Si se establece un corte vertical en la línea divisoria de los cuadrantes I y III, en el primer grupo de columnas, cuyo título es uso intermedio, se registra la utilización de los bienes y servicios por parte de un agente económico como insumo para su proceso productivo. El segundo grupo de columnas, cuyo título es uso final, muestra las compras por parte de los usuarios finales clasificadas por destino: consumo de los hogares (compras de bienes de consumo), consumo del gobierno (compra bienes de consumo colectivo en nombre de toda la sociedad), formación bruta de capital fijo (compras de activos no financieros producidos por parte de sociedades, gobierno u hogares productores), variación de existencias (bienes acumulados en inventarios) y exportaciones.

Ahora bien, respecto a la lectura por cuadrantes, el cuadrante I refleja las compra ventas con destino intermedio (para producir otros bienes y servicios). El cuadrante II muestra las compra ventas de usos de factores productivos necesarios para producir bienes y servicios: remuneraciones a los asalariados, superávit bruto de explotación e ingreso bruto mixto. El cuadrante III indica las compra ventas con destino final (consumo, inversión y exportación). La suma del cuadrante I y del cuadrante II, en sentido vertical, detalla el costo de la producción de los sectores industriales correspondientes. No obstante, es importante señalar que las actividades económicas generan productos principales (bienes característicos del sector) y productos secundarios (bienes típicos de otros sectores). En otras palabras, cada columna refleja los costos necesarios para producir tanto los bienes principales de la actividad como los productos secundarios.

Ahora bien, es importante destacar que en la matriz intersectorial de Tabasco el sistema de salud es concebido como un subconjunto del

\footnotetext{
${ }^{3}$ Catalogados según la clasificación del Sistema de Cuentas Nacionales (Eurostat et al. 1993).

${ }^{4}$ En las estimaciones realizadas para el sector salud se pudieron identificar únicamente las importaciones correspondientes a bienes, no así las de servicios de atención médica.
} 
sistema económico que incluye las unidades económicas involucradas en la producción, consumo y distribución de bienes y servicios de salud, contenidos en los sectores de servicios de la salud y administración pública. ${ }^{5}$ En consecuencia, las actividades que realizan en el marco de los servicios de salud, se entienden como aquellas acciones destinadas a mejorar las condiciones de vida de la población a través de la promoción, prevención, recuperación y rehabilitación de la misma.

En tanto las transacciones realizadas por las instituciones encargadas de la gestión administrativa del sector fueron clasificadas y valoradas como actividades de la administración pública que, por ende, quedaron fuera del sector salud. En particular, en este sector de actividades se reconocen cuatro de ellas relacionadas con la administración de la salud: administrativas federales de instituciones de bienestar social, administrativas estatales de bienestar social, administrativas federales de instituciones de seguridad social y actividades administrativas estatales de instituciones de seguridad social.

Finalmente, a efecto de comprender el alcance de la versión modificada del sistema de insumo-producto en Tabasco es necesario delimitar las acciones realizadas. En primer lugar, la matriz de demanda intermedia original era rectangular (16 X 19), por lo cual tuvimos que cambiar la matriz a una cuadrada (16 x16). Para ello, eliminamos las columnas hogares con servicios domésticos y diferencias. En segundo lugar, ajustamos los bordes de la matriz de transacciones totales. Por ello, existen algunas diferencias de menos de $3 \%$ con las estimadas por Armenta (2007).

\section{CUENTA SATÉLITE DE SALUD DE TABASCO}

Evaluar la efectividad del gasto público y privado en salud aporta los conocimientos necesarios para definir el comportamiento de las familias con respecto a sus gastos de salud, por lo tanto, diseñar un instrumento que amplíe la capacidad analítica del sector proporciona información intrasectorial.

\footnotetext{
${ }^{5}$ Es importante destacar que el sistema de salud que abarca las actividades cuya finalidad principal es promover, restablecer o mantener la salud, es el conjunto de personas, recursos y acciones cuyo propósito primario es mejorar la salud, así como las normas que regulan las actividades orientadas hacia ese fin (OMS, 2003).
} 
Asimismo, medir el valor agregado bruto, el consumo intermedio y el producto interno bruto del sector salud y vincular los flujos monetarios con el análisis de gasto por agentes permiten precisar un análisis del sector salud.

Desde un punto de vista conceptual, la cuantificación de la estructura del gasto de salud requiere considerar un sistema triaxial para el registro del gasto, según el cual se clasifica la atención de la salud por funciones, los sectores económicos proveedores de servicios de salud y las fuentes de financiamiento de la atención de la salud. Además de establecer los límites del sector de manera explícita, definiendo los contenidos de cada categoría, a manera de facilitar el seguimiento en el tiempo del comportamiento de los indicadores.

Estos conceptos han sido desarrollados ampliamente en el marco analítico de las Cuentas Satélites de Salud, además Tabasco cuenta con una estimación desarrollada en una tesis de maestría cuya construcción se basa en el método de recopilación de acuerdo con las recomendaciones del Sistema de Cuentas Nacionales de 1993 de Naciones Unidas y la Organización Panamericana de la Salud. En dicha cuenta se identificaron 12 servicios principales de la salud ${ }^{6}$ y se lograron estimar 4 actividades y servicios relacionados con la gestión administrativa de salud, ${ }^{7}$ entre las que destaca el Sistema de Seguro Popular (SSP) de la entidad. Adicionalmente, se pudieron integrar dos actividades complementarias de salud, como la de productos farmacéuticos y seguros médicos (Fuentes, 2010).

Con el fin de describir la demanda de productos por cada tipo de actividad principal del sector, ya sea intermedio o final, en Tabasco para 2003 se construyó una matriz, a partir de la Cuenta Satélite, que se nombró como: utilización de la salud (cuadro 3), la cual está construida a precios de

\footnotetext{
${ }^{6}$ Los 12 servicios principales de salud son: servicios hospitalarios generales (HG), servicios hospitalarios especiales (HE), servicios de consultas en medicina general (CG), servicios de consulta en medicina especial (CE), servicios de consulta en nutrición (CN), servicios de consulta odontológica (CO), servicios de medicina natural (MN), servicios de atención ambulatoria (SA), servicios de laboratorios y banco de órganos (L), centro de atención médica (CA), asistencia social (AS) y guarderías y cuidados infantiles (G). (Fuentes, 2010).

${ }^{7}$ Las 4 actividades-productos son: actividades administrativas federales de salud (AF), actividades administrativas estatales de salud (AE), actividades administrativas de instituciones de seguridad social federal (SSF) y actividades administrativas de instituciones de seguridad social estatal (SSE). (Fuentes, 2010).
} 
comprador. ${ }^{8}$ La matriz utilización de la salud posee una lógica horizontal y vertical, por lo tanto, su lectura se realiza por filas y por columnas. La lectura por fila muestra el destino (usos) de los productos (bienes y servicios), ya sea hacia la demanda intermedia (productos utilizados como insumos) o hacia la demanda final. La lectura por columna muestra la demanda de productos (o estructura de costes) por parte de las actividades económicas principales. La matriz se conforma por cuentas, a su vez, éstas se integran en tres subcuentas: usos intermedios (I), usos finales (II) y empleos de valor agregado (III) (ONU et al. 1993). En particular, los cuadrantes I y II, las filas de la parte superior reflejan la utilización de productos; ${ }^{9}$ y el cuadrante III la utilización de factores productivos empleados por cada actividad económica.

Las columnas detallan las compras por parte de las actividades. Si nos centramos en el cuadrante I, en el primer grupo de columnas, cuyo título es uso intermedio, se registra la utilización de los bienes y servicios por parte de los sectores económicos como insumo para su proceso productivo. Si nos centramos en el cuadrante II, las columnas, cuyo título es uso final, muestra las compras por parte de los usuarios finales clasificadas por destino: consumo de los hogares (compras de bienes de consumo) y consumo del gobierno (compra bienes de consumo colectivo). Respecto de la lectura por cuadrantes, el I refleja las compraventas con destino intermedio (para producir otros bienes y servicios del sector salud), el II indica las compraventas con destino final (consumo, inversión y exportación) y el cuadrante III muestra las compraventas de usos de factores productivos necesarios para producir bienes y servicios: remuneraciones a los asalariados, excedente bruto de explotación e ingreso bruto mixto..

\footnotetext{
${ }^{8}$ El precio al comprador es el precio pagado por el demandante, incluye márgenes de comercio y transporte e impuestos netos de subsidios sobre los productos. Mientras que el precio básico es el precio recibido por el productor, no incluye márgenes ni impuestos netos de subsidios sobre los productos.

${ }^{9}$ Los 20 productos son: alimentos y bebidas procesadas (ABP), productos farmacéuticos $(\mathrm{PF})$, Otros productos farmacéuticos ( $\mathrm{OPF}$ ), combustibles (Comb), maquinaria y equipo (MyE), papelería (Pap), productos de limpieza (PL), vestuario y prendas (VyP), equipo de transporte (ET), equipos médicos (EM), luz y agua (LyA), construcción (Const), restaurantes (Rest), telecomunicaciones (T), banca (B), servicios de alquiler (SA), servicios profesionales (SP), servicios de publicidad (SP), servicios de empaques (SEmp), otros servicios (OS) y pago de licencias (PLic). (Fuentes, 2010).
} 


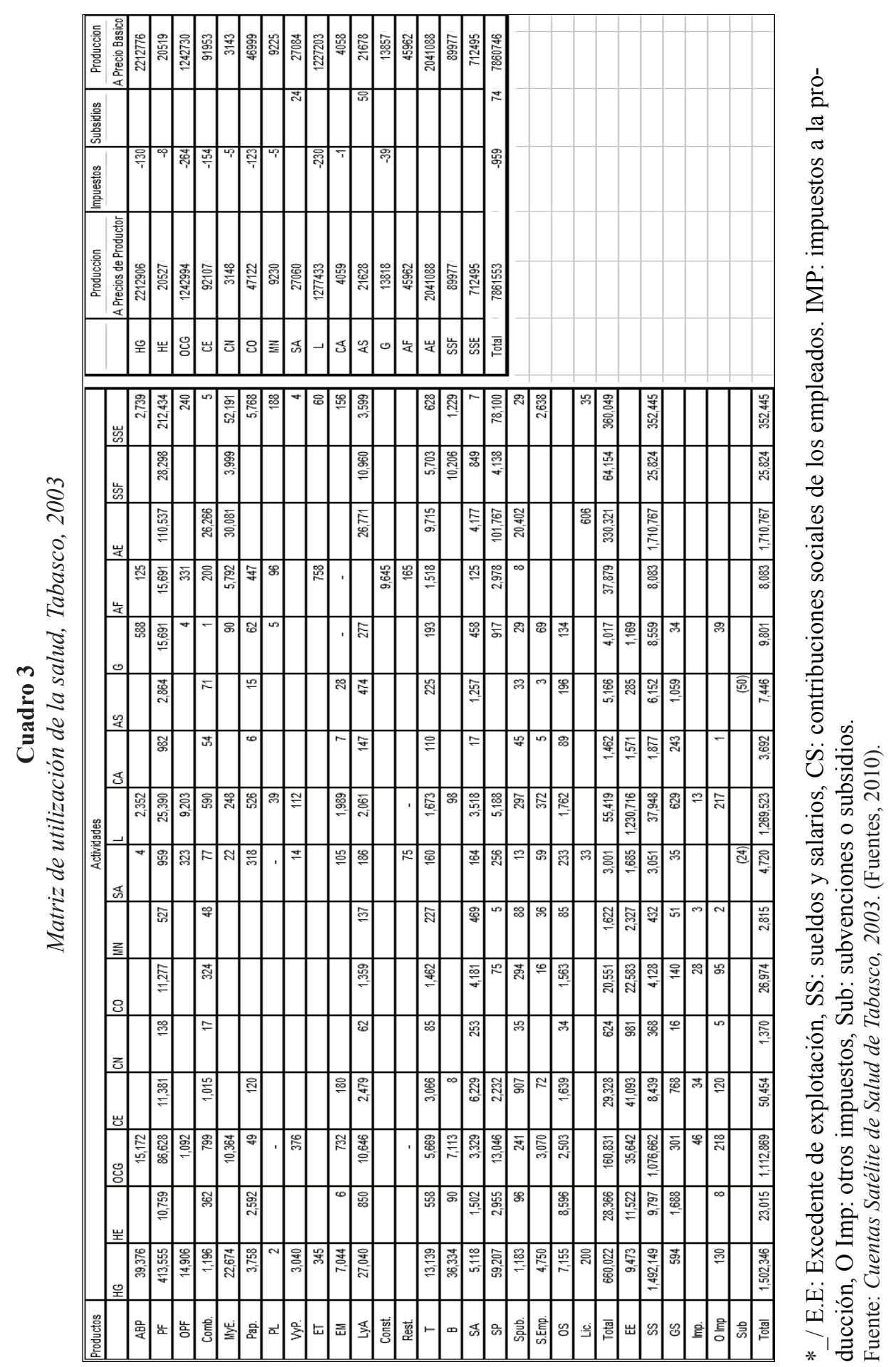

Revista de Economía - Vol. XXX - Núm. 80 
En la matriz de Utilización de la Salud del estado, en un sentido vertical, la suma del cuadrante I y II detalla el costo de la producción de la industria correspondiente. De manera complementaria a la matriz de Utilización se emplea la Matriz de Oferta de Salud, la cual reporta la producción total u oferta del sector de salud de Tabasco, al considerar las actividades económicas o servicios por salud (cuadro 4), esta matriz describe la oferta total a precios de productor de los servicios de salud y a precios básicos, es decir, el precio del productor menos los impuestos más subsidios.

Las actividades económicas de salud demandan productos principales (bienes característicos del sector) y productos secundarios (bienes típicos de otros sectores). Es preciso estimar una matriz de Producción de Salud para Tabasco (cuadro 5) que ofrezca información sobre las producciones, generar tanto las actividades principales del sector.

\section{Cuadro 4}

Matriz de oferta o producción total

\begin{tabular}{|c|r|r|r|r|}
\hline \hline & $\begin{array}{c}\text { Producción a precios de } \\
\text { productor }\end{array}$ & Impuestos & Subsidios & $\begin{array}{r}\text { Producción a precios } \\
\text { básicos }\end{array}$ \\
\hline \hline HG & $2,212,906$ & -130 & & $2,212,776$ \\
\hline HE & 20,527 & -8 & & 20,519 \\
\hline OCG & $1,242,994$ & -264 & & $1,242,730$ \\
\hline \hline CE & 92,107 & -154 & & 91,953 \\
\hline CN & 3,148 & -5 & & 3,143 \\
\hline CO & 47,122 & -123 & & 46,999 \\
\hline MN & 9,230 & -5 & & 9,225 \\
\hline SA & 27,060 & & 24 & 27,084 \\
\hline L & $1,277,433$ & -230 & & $1,277,203$ \\
\hline CA & 4,059 & -1 & & 4,058 \\
\hline AS & 21,628 & & & 21,678 \\
\hline \hline G & 13,818 & -39 & & 13,857 \\
\hline AF & 45,962 & & & 45,962 \\
\hline \hline AE & $2,041,088$ & & & $2,041,088$ \\
\hline SSF & 89,977 & & & 89,977 \\
\hline SSE & 712,495 & & & 712,495 \\
\hline OTAL & $7,861,553$ & -959 & & $7,860,746$ \\
\hline \hline
\end{tabular}

Fuente: Cuentas Satélite de Salud de Tabasco, 2003. (Fuentes, 2010). 


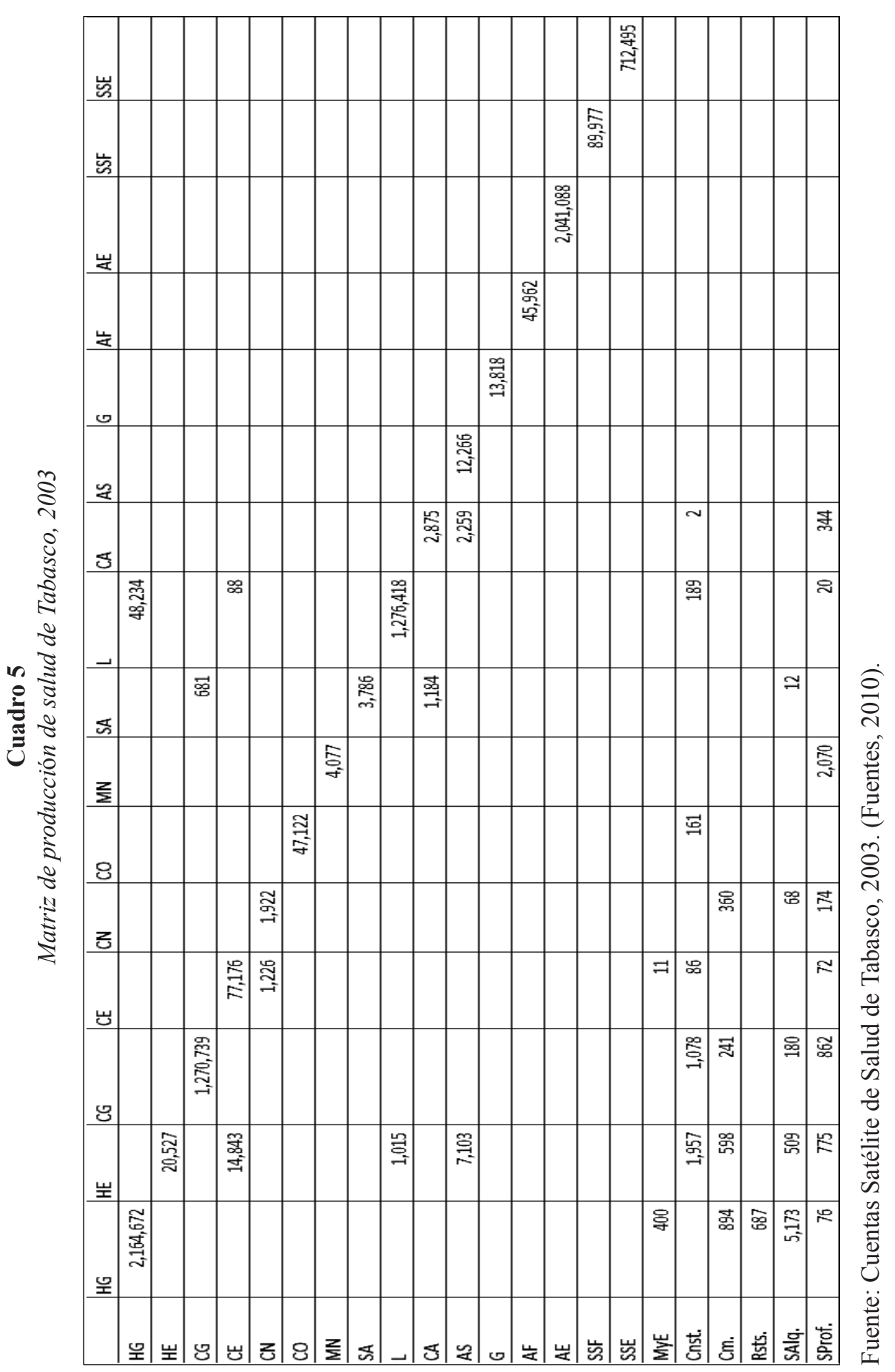

Revista de Economía - Vol. XXX - Núm. 80 


\section{EFECTIVIDAD DEL GASTO EN SALUD EN TABASCO}

Desde la perspectiva microeconómica el Seguro Popular de Salud tiene importantes implicaciones, entre las cuales podemos mencionar el aumento en la demanda de atención médica como consecuencia del subsidio a las familias, este hecho ejerce presión en los gastos de salud, por lo que es necesario aumentar la oferta de los servicios de salud debido al incremento en el número de usuarios.

Puesto que la Cuenta Satélite de Salud de Tabasco brinda información sobre las estructuras de gasto de los prestadores públicos y privados, se pueden conocer cuáles son los bienes y servicios incluidos en la función de producción, así como las fuentes de financiamiento y demandas de insumos por parte de las actividades de salud.

\subsection{Quién gasta en salud}

La matriz de usos o utilización final permite determinar el gasto en salud a partir del usuario final. Estos usuarios pueden caracterizarse como provenientes del sector gobierno general, en cualquiera de sus niveles, y de los hogares como consumo individual. También pueden incluirse el gasto del exterior, en formación bruta de capital fijo, y las variaciones de existencias, que son componentes de la demanda final del gasto en salud. Por consiguiente, el gasto del sector de salud de Tabasco, se considera el gasto en salud por usuario final, se reporta en el cuadro 6, en donde observamos que el consumo colectivo de gobierno constituyó $79 \%$ del total del gasto en servicios de salud, mientras el restante $21 \%$ fue consumo individual de los hogares.

\section{Cuadro 6}

Gasto en salud por usuario final, 2003

\begin{tabular}{|c||r||r||r|}
\hline & Monto & \% s/gasto total & \% s/PIB \\
\hline \hline Hogares & 1,663445, & 21.15 & 2.14 \\
\hline \hline Gobierno & $6,247,445$ & 79.46 & 8.06 \\
\hline
\end{tabular}

Fuente: Cuenta Satélite de Salud de Tabasco (Fuentes, 2010). 


\subsection{En qué actividades se gasta en salud}

Con el fin de saber cuáles son las actividades en las que se gasta se construye la Matriz de Oferta, misma que describe como las unidades de salud en sus actividades demandan insumos. En el caso de la Cuenta Satélite de Salud de Tabasco hay 12 actividades principales (diagonal principal) que dan como resultado la producción del sector de la salud. Por otro lado, existen adicionalmente actividades secundarias de salud que están vinculadas indirectamente con los servicios de alquiler de alguna parte del inmueble de la unidad de salud, así como también quienes prestan servicios profesionales a otras instituciones y comercio de ciertos bienes. Destacan las actividades de hospitales especializados, laboratorios y asistencia social. El gasto del sector de la salud por tipo de servicio o actividad económica en la entidad federativa se muestra en el cuadro 7.

\section{Cuadro 7}

Gasto de los hogares en salud por tipo de servicio, 2003

\begin{tabular}{||c||r||r||r|}
\hline & \multicolumn{1}{|c|}{ Monto } & \% s/gasto total & \multicolumn{1}{c|}{$\%$ s/PIB } \\
\hline \hline HG & 123,760 & 7.43 & 0.15 \\
\hline \hline HE & 22,225 & 1.33 & 0.028 \\
\hline \hline CG & 221,873 & 13.33 & 0.28 \\
\hline \hline CE & 93,645 & 5.62 & 0.12 \\
\hline CN & 3,148 & 0.18 & 0.40 \\
\hline \hline CO & 47,122 & 2.83 & 0.06 \\
\hline \hline MN & 9,230 & 0.55 & 0.01 \\
\hline \hline SA & 24,965 & 1.50 & 0.03 \\
\hline \hline L & $1,077,075$ & 64.74 & 1.39 \\
\hline \hline CA & 4,059 & 0.24 & 0.52 \\
\hline \hline AS & 21,628 & 1.30 & 0.02 \\
\hline \hline G & 14,716 & 0.88 & 0.01 \\
\hline \hline Total & $1,663,445$ & \multicolumn{2}{|c|}{} \\
\hline \hline
\end{tabular}

Fuente: Cuenta Satélite de Salud de Tabasco (Fuentes, 2010). 
Se concluye que los hogares realizaron un mayor consumo en laboratorios (L) con 65\%, 13\% se gastó en consultas generales (CG), los servicios de hospitalización general (HG) constituyeron 7\%, la consulta con especialistas (CE) $6 \%$ y los restantes servicios se encuentran en un rango de 1 a 3 por ciento. En el caso del gobierno su mayor gasto fue en la administración de servicios de hospitalización (AS) con 34\%, mientras que los servicios de hospitalización general (HG) fueron 33\%. Los servicios de consulta general (CG) significaron $17 \%$ y $11 \%$ se gastó en la administración de la seguridad social estatal.

\subsection{En qué producto se gasta}

La matriz de usos intermedios de Tabasco se agrupo en 20 bienes principales de la economía del estado, de esta forma permite determinar cuáles son los insumos que requieren las actividades económicas del sector salud. Estos bienes principales se pueden caracterizar como provenientes de las demandas de insumos por parte de las actividades económicas del sector salud. En el cuadro 8 se presentan los insumos vitales que se emplean, en promedio, para su operación por las actividades económicas del sector salud.

\section{Cuadro 8}

Gasto en salud por producto intermedio, 2003

\begin{tabular}{|c||r||r||r|}
\hline & Monto & $\%$ s/gasto total & $\%$ s/PIB \\
\hline \hline PF & 932610 & 53.51 & 1.20 \\
\hline \hline SP & 270864 & 15.54 & 0.34 \\
\hline \hline MyE & 125461 & 7.19 & 0.16 \\
\hline \hline LyA & 87048 & 4.99 & 0.11 \\
\hline \hline ABP & 60356 & 3.46 & 0.07 \\
\hline \hline PL & 330 & 0.01 & 0.0042 \\
\hline \hline T & 44131 & 0.25 & 0.056 \\
\hline \hline B & 55078 & 3.16 & 0.07 \\
\hline \hline OPF & 26099 & 1.49 & 0.033 \\
\hline \hline VyP & 3546 & 0.20 & 0.0045 \\
\hline \hline SA & 25943 & 1.48 & 0.033 \\
\hline SP & 23700 & 1.35 & 0.030 \\
\hline \hline
\end{tabular}

Fuente: Cuenta Satélite de Salud de Tabasco (Fuentes, 2010). 
Es evidente que el mayor gasto en salud corresponde a productos farmacéuticos con 53\%, el segundo lugar lo ocupan los servicios profesionales con $15 \%$, en tanto que $7 \%$ es destinado a la compra de maquinaria y equipo para la producción y $3 \%$ a las compras de alimento y bebidas procesadas y los servicios de banca e intermediación financiera. En cuanto a qué actividad es la que más gasta en insumos tenemos que la de hospitales generales y los servicios administrativos de la seguridad estatal, mientras que en un tercer sitio se encuentra la administración de salud estatal.

\subsection{Cómo se financia la salud}

En el sistema central, es decir, en el sistema intersectorial para Tabasco, las transacciones realizadas por las instituciones encargadas de la gestión administrativa del sector salud fueron clasificadas y valoradas como actividades de la administración pública y quedan fuera del sector salud. Sin embargo, en el sistema de la Cuenta Satélite de Salud de Tabasco estas fueron clasificadas en cuatro grandes grupos: actividades administrativas federales de instituciones de bienestar social, actividades administrativas estatales de instituciones de bienestar social, actividades administrativas federales de instituciones de seguridad social y actividades administrativas estatales de instituciones de seguridad social. ${ }^{10}$

De este modo, el financiamiento y la provisión de servicios de salud pública en Tabasco se organizan a través de dos agentes: estatal y federal. En el cuadro 9 se presentan las 4 actividades administrativas que representan el flujo de financiamiento del sector salud.

\footnotetext{
${ }^{10}$ Este ejercicio requirió la reclasificación como actividades de salud. Cada una tiene importancia por sí misma, puesto que se refieren a actividades que aportan una gran cantidad de producción/gastos, consumo intermedio y empleo en la Cuenta Satélite de Salud (Fuentes, 2010: 70).
} 


\section{Cuadro 9}

Gasto en salud por fuente de gasto, 2003

\begin{tabular}{|c||r|r||r|}
\hline \hline & Monto & $\%$ s/gasto total & $\%$ s/PIB \\
\hline \hline Administración federal de la salud (SPS) & 45,962 & 1.59 & 0.059 \\
\hline \hline Administración estatal de salud (AES) & $2,041.088$ & 70.63 & 2.63 \\
\hline \hline Administración de seguridad social federal (AFSS) & 89,977 & 3.11 & 0.11 \\
\hline \hline Administración de seguridad social estatal (AESS) & 712,495 & 24.65 & 0.92 \\
\hline \hline
\end{tabular}

Fuente: Cuenta Satélite de Salud de Tabasco (Fuentes, 2010).

Debido a que en la Cuenta Satélite de Tabasco el Programa del Seguro Popular de Salud se ubica dentro de las actividades de Administración Federal de la Salud, en esta actividad sólo fue posible identificar el gasto realizado en el programa, sin identificar mayores detalles que permitieran diferenciar el gasto administrativo del gasto en técnica médica. Por lo tanto fue agrupado en la primer categoría de actividades de administración pública (Fuentes, 2010).

En cuanto a las demandas de insumos intermedios que generó este gasto, la mayor parte se refirió a productos farmacéuticos con $41 \%$, servicios de edificación y construcción con $25 \%$, respectivamente, y compras de maquinaria y equipo con $15 \%$. Adicionalmente, se destinó a servicios profesionales $8 \%$ y a telecomunicaciones $4 \%$. El resto de los insumos demandados oscilan entre 1 y $2 \%$ (Fuentes, 2010).

En el estado de Tabasco la afiliación al programa ascendió a 87, 846 familias (SSA, 2004); cada unidad familiar costó \$7,727.46 pesos de 2002, que incluye medicamentos, servicios médicos y administrativos, en tanto que el programa recibió un subsidio de $\$ 2,100$ pesos y una cuota de 204 pesos por familia (Santos y Martínez, 2005). De acuerdo con el estudio de la Cuenta Satélite de Salud, el costo por familia afiliada fue de $\$ 523$ pesos. Además a nivel nacional, según la Secretaría de Salud Federal, el segundo mayor presupuesto público ejercido (ibídem).

Un fenómeno observable en Tabasco fue que la población que carecía de seguridad social sustituyó la demanda de servicios privados por demanda del Seguro Popular de Salud (Garcíay Tejero, 2003). En consecuencia, el 
resultado esperado fue la caída de sus gastos catastróficos, ${ }^{11}$ por lo menos en aquellos servicios de salud asociados al cuadro básico del programa, lo que aumentó los gastos a otras necesidades. Por ello el subsidio federal ha sido eficaz, en la medida que a las familias afiliadas les otorga cierta protección financiera.

Dentro de la Cuenta Satélite de Tabasco, las actividades realizadas por la Secretaría de Salud del estado y por el Centro Estatal de Hemoterapia están dentro de la actividad de Administración Estatal de Salud. Esta actividad es central debido a que la mayor parte del presupuesto de la Secretaría se emplea en las compras de las unidades de salud. En cuanto a la demanda de insumos intermedios derivados del gasto de la actividad tenemos que la mayor parte recayó en productos farmacéuticos con 33\%, le siguió servicios profesionales con $31 \%$ y compras de maquinaria y equipo con 9 por ciento. Adicionalmente, destinó a combustibles y pago de electricidad y agua potable 8 por ciento. Otros con menor proporción fueron servicios de publicidad y telecomunicaciones con 6 y 3\%, respectivamente (Fuentes, 2010).

En el estado de Tabasco la población usuaria de servicios de salud registrados en la Secretaría fue de 1,074,666 personas, respecto del gasto en salud, según la Cuenta Satélite nos da un costo por usuario de \$1,899 pesos promedio.

Dentro de la Cuenta Satélite de Tabasco la gestión administrativa que provee servicios de seguridad social en el estado - Instituto de Seguridad y Servicios Sociales de los Trabajadores del Estado (ISSSTE), Instituto Mexicano del Seguro Social (IMSS) y Petróleos Mexicanos (PEMEX)-, cae en la actividad de Administración de Seguridad Social Federal.

En cuanto al gasto de esta actividad por institución tenemos que PEMEX participó con 52\%, mientras que el IMSS contribuyó con $40 \%$ y el ISSSTE con 8 por ciento. Por lo que hace a la categoría administrativa, el gasto de las tres instituciones respecto al total del gasto administrativo sólo representó $1.44 \%$. (Fuentes, 2010).

En relación con el aumento de la demanda de insumos intermedios, derivados del gasto de la actividad, tenemos que fue de $44 \%$ en productos

\footnotetext{
${ }^{11}$ De acuerdo con la CNPSS (2009) se consideran gastos catastróficos aquellas enfermedades que implican un alto costo en virtud de su grado de complejidad o especialidad y el nivel de frecuencia con que ocurren.
} 
farmacéuticos, $17 \%$ para el pago de suministro de electricidad y agua y $16 \%$ para los servicios financieros de la Banca. El resto de insumos en que incurren estas instituciones son telecomunicaciones, servicios profesionales y maquinaria y equipo.

Finalmente, en la Cuenta Satélite de Tabasco las actividades realizadas por el Instituto de Seguridad Social del Estado de Tabasco (ISSET) se incluyen como parte de la Administración de Seguridad Social del Estado. El gasto en insumos intermedios de esta actividad incluye productos farmacéuticos con $59 \%$, servicios profesionales con $22 \%$, maquinaria y equipo con $14 \%$ y el resto de las actividades, que varían entre uno y tres por ciento.

En el estado de Tabasco la población usuaria que demandó este servicio fue de 144, 729 personas, equivalente a $79 \%$ de la población reportada como derechohabiente. Adicionalmente, respecto al gasto del ISSSTE se tiene que operativamente costó $\$ 4,920$ pesos por cada usuario, siendo el primer lugar en costo por usuario entre los servicios administrativos de todas las instituciones.

\section{IMPACTO ECONÓMICO DEL GASTO DEL SEGURO POPULAR DE SALUD EN TABASCO}

Dadas las profundas implicaciones económicas y sociales de la puesta en marcha del SPSS y de su impacto directo, indirecto e inducido en Tabasco, el análisis subsecuente se desarrollará bajo la perspectiva del multiplicador sectorial del modelo de insumo-producto abierto. ${ }^{12}$

12 Los multiplicadores es un concepto pilar en el análisis macroeconómico definido "cómo un aumento en algún componente de la demanda agregada genera un aumento en el producto, ingreso o empleo mayor que el aumento original" y estos se clasifican como multiplicadores de producto, ingreso y empleo. A su vez, los multiplicadores del ingreso y del empleo se subdividen en dos tipos: aquéllos que consideran únicamente los efectos directos e indirectos de los cambios en cualquiera de los componentes de la demanda agregada (modelo cerrado) y aquellos que, además incluyen el impacto sobre el ingreso

y empleo, inducidos por cambios en el consumo (modelo abierto). En otras palabras, los multiplicadores del Tipo II miden los impactos directos, indirectos e inducidos sobre el ingreso y empleo ocasionados por un cambio en la demanda final, excluyendo de ésta el consumo (Fuentes y Cárdenas, 2010: 12). 
Hasta el momento se han planteado el origen y los fundamentos sociales y la efectividad del gasto del programa, así como de sus objetivos, instrumentación y beneficios médicos. Sin embargo, es importante hacer un abordaje del mismo desde su aporte a la economía regional, puesto que los impactos de su aplicación se reflejaran necesariamente en mayores indicadores estatales de producción, ingreso y empleo. En este sentido, el papel que tiene el Seguro Popular de Salud en Tabasco como instrumento de financiamiento de salud de las familias es de gran importancia económica ya que, por un lado, su aplicación tiene un reflejo inmediato en un aumento de la demanda de atención médica debido a la transferencia de subsidio público federal y, por el otro, evita el desembolso familiar que de otra manera tendrían que hacer para satisfacer sus necesidades de salud y pueden destinar dichos recursos al consumo de otros bienes y servicios, lo que produce un aumento en la demanda. En efecto, es la multiplicación dentro de la economía regional que, en el corto plazo, contribuirá a lograr un mayor crecimiento económico y social.

Como se estableció anteriormente dentro de la Cuenta Satélite de Salud de Tabasco, el Seguro Popular reportó un gasto directo por parte del gobierno federal y estatal que se muestra en el cuadro 10. Del total del gasto, el consumo intermedio, es decir, la utilización intermedia requerida para que esta actividad operara en el estado fue de $82 \%$, el restante $18 \%$ se destinaron al pago de sueldos y salarios.

\section{Cuadro 10}

Gasto en salud por el Programa del seguro popular, 2003

\begin{tabular}{|c|r|r|r|}
\hline & \multicolumn{1}{|c|}{ Monto } & $\%$ s/gasto total & $\%$ s/PIB \\
\hline Producción principal & 45,962 & 50 & 0.05 \\
\hline Valor agregado & 8,083 & 8.79 & 0.01 \\
\hline Consumo intermedio & 37,879 & 41.20 & 0.048 \\
\hline
\end{tabular}

Fuente: Cuenta Satélite de Salud de Tabasco. (Fuentes, 2010: 71).

La técnica de insumo-producto fue diseñada para analizar los impactos por sector económico de cambios exógenos en los componentes de la demanda final, en este caso el análisis de los cambios en el gasto de gobierno en salud, y nos permite computar tantos multiplicadores como sectores 
haya incluidos en la matriz de transacciones intersectoriales de Tabasco. El cuadro 11 muestra los multiplicadores de producción, ingreso y empleo para el estado de Tabasco, estos últimos se presentan en las versiones Tipo I y II; es decir, aquéllos multiplicadores que consideran simplemente los efectos directos e indirectos de los cambios en cualquiera de los componentes de la demanda agregada y, por otro lado, los multiplicadores que miden los impactos directos, indirectos e inducidos sobre el ingreso y empleo ocasionados por un cambio en la demanda final, excluyendo de ésta el consumo.

\section{Cuadro 11}

Multiplicadores sectoriales para Tabasco, 2003

\begin{tabular}{|c|c|c|c|c|c|}
\hline Sectores & Producción & \multicolumn{2}{|c|}{ Ingreso } & \multicolumn{2}{c|}{ Empleo } \\
\hline Agricultura y ganadería & & Tipo I & Tipo II & Tipo I & Tipo II \\
\hline Pesca y acuacultura & 1.184 & 1.760 & 2.100 & 1.650 & 1.930 \\
\hline Minería & 1.734 & 1.180 & 1.250 & 1.080 & 1.120 \\
\hline Alimentos, bebida y tabaco & 1.450 & 1.250 & 1.370 & 1.690 & 1.970 \\
\hline Otras manufacturas & 1.039 & 1.600 & 1.840 & 1.210 & 1.300 \\
\hline Servicios electricidad y agua & 1.268 & 1.410 & 1.560 & 1.690 & 1.930 \\
\hline Construcción & 1.606 & 1.060 & 1.080 & 1.700 & 1.980 \\
\hline Servicios de comercio y repara. & 1.522 & 1.210 & 1.290 & 1.310 & 1.420 \\
\hline Hoteles y restaurantes & 1.768 & 1.470 & 1.650 & 1.410 & 1.600 \\
\hline Transporte y conexos & 1.635 & 1.150 & 1.210 & 1.080 & 1.120 \\
\hline Servicios financieros y banca & 1.527 & 1.070 & 1.090 & 1.110 & 1.160 \\
\hline Servicios mobiliarios e inmob. & 1.318 & 1.150 & 1.220 & 1.300 & 1.410 \\
\hline Administración pública & 1.257 & 1.240 & 1.330 & 1.130 & 1.180 \\
\hline Servicios de educación & 1.067 & 1.210 & 1.300 & 1.090 & 1.130 \\
\hline Servicios de salud & 1.137 & 1.150 & 1.220 & 1.080 & 1.120 \\
\hline Servicios comunitarios & 1.535 & 1.200 & 1.280 & 1.240 & 1.320 \\
\hline Promedio total & 1.478 & 1.278 & 1.391 & 1.333 & 1.469 \\
\hline
\end{tabular}

Fuente: Elaboración propia. 
La interpretación de cualquier multiplicador monetario - producción e ingreso - es la siguiente: En el sector Salud por ejemplo, cada $\$ 1,000,000$ de pesos de incremento en el gasto de gobierno se genera una producción total con valor de $\$ 1,137,000$ pesos. O visto de otra forma, los multiplicadores indican que en el sector Salud, cada $\$ 1,000,000$ de pesos de incremento de la demanda final genera producción total con valor de \$1,137, 000 pesos. Los multiplicadores de ingreso tipo II indican que los hogares del sector recibirán ingresos de aproximadamente $\$ 1,220$, 000 por cada millón de pesos de aumento en la demanda final; en el caso del multiplicador del empleo es lo siguiente; En el sector salud el aumento de $\$ 1,000,000$ de pesos en el sector genera 11.20 empleos.

En el contexto específico del estudio se tiene que el gobierno estatal por concepto del programa del Seguro Popular de Salud dispone de $\$ 45,962,000$ pesos. Entonces ¿cuál es el impacto económico de este gasto de gobierno exógeno en la producción, ingreso y empleo de Tabasco? Para medir el impacto necesitamos conocer la composición de la demanda final del gasto por concepto del programa de Seguro Popular de Salud. Una propuesta es considerar la estructura de gasto de insumos intermedios que aparecen explícitamente en la Cuenta Satélite de Salud de Tabasco. ${ }^{13} \mathrm{La}$ estructura de gasto del programa del Seguro Popular de Salud por tipo de insumo intermedio y actividad económica que demanda en la entidad federativa se muestra en el cuadro 12.

\section{Cuadro 12}

Gasto en salud por tipo de servicio y fuente de financiamiento, 2003

\begin{tabular}{|c|r|r|r|r|}
\hline Sectores & $\begin{array}{c}\text { Demanda } \\
\text { intermedia } \\
\text { SSP }\end{array}$ & $\begin{array}{c}\text { Demanda } \\
\text { intermedia } \\
\text { AES }\end{array}$ & $\begin{array}{c}\text { Demanda } \\
\text { intermedia } \\
\text { AFSS }\end{array}$ & $\begin{array}{c}\text { Demanda } \\
\text { intermedia } \\
\text { AESS }\end{array}$ \\
\hline Agricultura y ganadería & 0 & 0 & 0 & 0 \\
\hline Pesca y acuacultura & 0 & 0 & 0 & 0 \\
\hline Minería & 0 & 0 & 0 & 0 \\
\hline Alimentos, bebida y tabaco & 1,379 & 0 & 0 & 0 \\
\hline
\end{tabular}

${ }^{13}$ Para extender el análisis a la erogación pública en materia de salud se amplía en el cuadro 12 el gasto de salud por tipo de servicio y fuente de financiamiento de Tabasco. 


\begin{tabular}{|c|r|r|r|r|}
\hline Otras manufacturas & 25,739 & 81,644 & 2,699 & 85,499 \\
\hline Servicios electricidad y agua & 0 & 673,559 & 39,590 & 420,372 \\
\hline Construcción & 11,491 & 163,287 & 15,296 & 14,250 \\
\hline Servicios de comercio y repara & 5,056 & 0 & 0 & 0 \\
\hline Hoteles y restaurantes & 0 & 244,931 & 10,797 & 0 \\
\hline Transporte y conexos & 1,838 & 0 & 0 & 0 \\
\hline Servicios financieros y banca & 0 & 61,233 & 2,699 & 0 \\
\hline Servicios mobiliarios e inmob. & 0 & 0 & 14,396 & 35,625 \\
\hline Administración pública & 0 & 0 & 0 & 0 \\
\hline Servicios de educación & 0 & 0 & 0 & 0 \\
\hline Servicios de salud & 3,217 & 61,233 & 0 & 0 \\
\hline Servicios comunitarios & 3,677 & 632,737 & 2,699 & 156,749 \\
\hline Total & 45,962 & $2,041.088$ & 89,977 & 712,495 \\
\hline
\end{tabular}

Fuente: Elaboración propia a partir de Fuentes (2010).

Así, en el año 2002, al partir de un gasto del Seguro Popular de Salud agregado de 45,962, 000 de pesos se generó un efecto directo de 19,079,370 pesos en la economía de Tabasco. A partir de la distribución la demanda intermedia de los principales sectores de la economía, incluidos en la Cuenta Satélite en Salud de Tabasco, se analizan los impactos de tipo I y II del SPSS en la economía de la región, donde destacan seis sectores además del sector salud. El sector de alimentos, bebidas y tabaco destaca en el impacto del ingreso del tipo II, el cual asciende a 1,158.36 millones de pesos; ahora bien, por el lado del impacto en el empleo el sector que se adjudicó un mayor impacto tipo II fue la construcción con 11,261 empleos, esto debido a la inversión en infraestructura destinada al mejoramiento o aumento del número de unidades de salud en Tabasco.

\section{CONCLUSIONES}

La Cuenta Satélite de Salud de Tabasco es un marco analítico en el que se considera explícitamente, si bien de forma agregada, el papel de la 
erogación pública y privada en materia de salud. En particular, en ella podemos observar que el gasto público en servicios de salud fue cinco veces mayor que el gasto realizado por los hogares en 2003. Igualmente, notamos que, en su mayoría, este gasto se realizó en hospitalización general, le siguieron las consultas generales, que correspondieron a las jurisdicciones municipales de salud, y por último aparece el gasto en laboratorios, según el gasto que erogó el gobierno en la economía regional. Lo anterior sugiere que las familias afiliadas no desembolsan parte de sus ingresos en servicios de costos altos, como es la hospitalización.

Relativo al gasto público en servicios administrativos, es evidente el tipo de actor que representa el Seguro Popular al ser un eje de la última reforma de salud cuyo objetivo ha sido equilibrar el financiamiento de la salud. Reportó 84,846 familias que le costaron a la administración operativa 240 pesos por familia afiliada. Se considera se amplié y disminuya el costo hacia el futuro, además que se espera que se beneficie el bolsillo de los hogares al dejar de consumir servicios de salud de índole privada, sobre todo en el caso de las familias más pobres.

En cuanto a las demandas de insumos intermedios que generó el SPSS se reporta que la mayor parte se refirió a productos farmacéuticos con $41 \%$, servicios de edificación y construcción $25 \%$ y, compras de maquinaria y equipo con 15 por ciento. Adicionalmente, destinó a servicios profesionales $8 \%$ y a telecomunicaciones $4 \%$, el resto de los insumos demandados por el programa oscilan entre uno y dos por ciento.

Por otro lado, con el objetivo de valorar si dicho impacto es elevado o no, se aplicó la metodología insumo-producto. Con el modelo abierto de insumo-producto encontramos que a partir de un gasto inicial del Seguro Popular de Salud de $\$ 45,962,000$ de pesos se generó un efecto directo de 19,079,370 de pesos en la economía de Tabasco. Mientras que, si se cierra el modelo de insumo producto considerando los hogares como endógenos, es estimable que el efecto directo e indirecto de producción en este sector represente $\$ 6,296.79$ millones de pesos, en tanto que el impacto tipo II en el ingreso alcanza un monto de $\$ 10,111.64$ millones de pesos y en el empleo se generaron 5,515.44.

En general, algunos de los resultados presentados sugieren que los impactos directo e indirecto del sector de la salud sobre la economía regional 
son relativamente elevados, debido a que el consumo de servicios asistenciales de salud normalmente tiene un alto contenido local. De modo que, la nueva demanda final de servicios de salud, debe ser satisfecha de forma creciente con servicios locales. Finalmente, es necesario insistir en que el impacto económico de los servicios de salud sobre Tabasco es, básicamente, el resultado de aprovechar las ventajas competitivas de la economía local, por ello, la valoración de ampliar el SPSS debe hacerse en un contexto integral que considere no sólo lo indicadores de salud, consistencia y resultado, sino también su impacto económico regional, pues provee en las regiones un motor endógeno de competencia. 


\section{BIBLIOGRAFÍA}

Ángeles Sevilla, Alejandro et. al. 2011. El sistema de salud en México perspectiva histórica, evolutiva y de globalización, Instituto de Investigación Económica y Social Lucas Alamán, A.C., México. Armenta, Aida. 2007. Modelo insumo-producto para Tabasco (integración de la matriz de insumo-producto), FOMIXTAB-2003-c01-9544, Universidad Juárez Autónoma de Tabasco.

Andreff, Wellis. 2001. "Los intentos europeos y franceses de elaborar una contabilidad nacional de la economía del deporte", en J.M. Otero (comp.), Incidencia económica del deporte, Instituto Andaluz del Deporte, Málaga, pp. 23-53.

Comisión Nacional de Protección Social en Salud, 2010, sección Seguro Popular, consultado en: < http://www.seguropopular.salud.gob. $\mathrm{mx} /$ index.php?option $=$ com_content $\&$ view $=$ article $\& i d=272 \&$ Ite mid=286 $>$ Fecha de consulta:2013

Comisión Nacional de Protección Social en Salud, 2009, Fondo de Protección contra Gastos Catastróficos/Sistema de Protección Social en Salud, consultado en: <http://www.seguro-popular.salud.gob. $\mathrm{mx} /$ index.php?option $=$ com_content $\&$ view $=$ article \&id $=84$ :des cripcion \&catid $=34$ :fondoprotecciongastoscatastroficos-\&Item id=177> Fecha de consulta: Febrero de 2013

Eurostat, FM1, OCDE,,ONU y BM.1993. Sistema de Cuentas Nacionales 1993, Bruselas, París Luxemburgo, Nueva York y Washington.

Fuentes Castillo, Martha Elena. 2010. Metodología y análisis de la cuenta satélite de salud: estimulación para el estado de Tabasco, tesis de Maestría, Universidad Autónoma de Coahuila,.

Fuentes, Noé y Ana Cárdenas. 2010. "Evaluación del impacto de alternativas e utilización de los excedentes petroleros sobre la economía mexicana: Una aplicación del modelo insumo-producto", Revista Economía Mexicana, Nueva Época, vol. 19, núm. 2, pp. 379-399. García Rodriguez J y Tejero Vera, A. (2003) "Programa del Seguro Popular de Salud en México: Un análisis económico" Salud en Tabasco 9(001), 178-187 
Hernández, Raúl. 2004. El impacto del turismo sobre las importaciones: Aspectos metodológicos y empíricos, trabajo presentado en las I Jornadas de Economía del Turismo, Facultad de Ciencias Económicas y Empresariales, Universitat de les Illes Balears (mimeo).

Halba, Bill. 1997. Économie du sport, Economica, París.

Heinemann, Keith. 1998. Introducción a la economía del deporte, Paidotribo, Barcelona.

Instituto Nacional de Estadística y Geografía, 2000, Censo de Población y Vivienda 2000, México, D.F

Késenne, Suzane. 2001. "El problema de los estudios de incidencia económica en el deporte", en, J.M. Otero (comp.), Incidencia económica del deporte, Instituto Andaluz del Deporte, Málaga, pp. 91-99.

Rangel, Gudelia y Martínez Pellégrini, S. 2007. Seguro Popular una evaluación del desempeño de los sistemas estatales de salud. Descentralización y calidad de vida, Colegio de la Frontera Norte, México (mimeo).

Moati, Pierre. 1990. La filière sport: approche économique d'un marché en mutation, CREDOC, París.

Otero, José $\mathrm{M}^{\mathrm{a}}$. (comp.) et al. 2000. Estudio socioeconómico del deporte en Andalucía 1998-1999, Junta de Andalucía, Consejería de Turismo y Deporte, Sevilla.

Organización Mundial de la Salud (OMS). 2000. Informe sobre la salud en el mundo, 2000. Mejorar el desempeño de los sistemas de salud, $<$ www.who.int/whr/2000/es/>.

Santos M. y Martinez J. (2005) "Gastos Privado en Salud en Tabasco" Salud Publica de México 47 (suplemento 1).

Secretaría de Salud, 2002, Reglas de Operación e indicadores de gestión y evaluación del Seguro Popular de Salud, Diario Oficial de la Federación. México, D.F.

Secretaría de Salud, 2004, Situación de Salud en México 2003: Indicadores Básicos. Secretaría de Salud, México.

Secretaría de Salud, 2008, Modelo iIntegrador de aAtención a la Salud, Segunda edición, México, D.F. pág. 64. 
United Nations, OECD, WTO \& European Commission. 2001. Tourism Satellite Account: Recommended Methodological Framework, OECD, New York.

Villalba Caballo, F (dir) et al. 2002, Deporte y economía: una cuantificación de la demanda deportiva en Andalucía, Analistas Económicos de Andalucía, Málaga. 